\title{
DECOMPOSITION OF PRODUCTS OF MODULAR REPRESENTATIONS ${ }^{1}$
}

\author{
BY THOMAS RALLEY
}

\author{
Communicated by I. Reiner, June 24, 1966
}

Let $G$ be a cyclic group of order $p^{N}$ with generator $g, p$ a prime, and let $K G$ be the group algebra over a field $K$ of characteristic $p$. Green [1] and Srinivasan [3] gave formulas for the decomposition of tensor products of $K G$-modules into direct sums of indecomposables. We outline here an alternative procedure, based on the theory of elementary divisors, for obtaining these formulas.

For each $r, 1 \leqq r \leqq p^{N}$, there is an indecomposable $K G$-module of dimension $r$. It affords a matrix representation $g \rightarrow V_{r}=E_{r}+H_{r}$, where $E_{r}$ is the $r \times r$ identity matrix and $H_{r}$ is the $r \times r$ matrix with ones along the superdiagonal and zeros elsewhere. The characteristic matrix $V_{r}-\lambda E_{r}$ has exactly one elementary divisor, $(1-\lambda)^{r}$. Thus the decomposition of a $K G$-module can be determined from knowledge of the elementary divisors of the matrix representation which it affords.

Lemma ([2]). The elementary divisors of $V_{m} \otimes V_{n}-\lambda E_{m n}$ are the same as those of $M=\left[H_{m}+(1-\lambda) E_{m}\right]^{n}$.

Put $t=1-\lambda$. Expansion by the binomial theorem shows that $M$ is an upper triangular matrix with $(i, j)$ entry $a_{i j}=C(n, j-i) t^{n-j+i}$, $1 \leqq i, j \leqq m$, where the binomial coefficient $C(n, j-i)$ is to be regarded as an element of $K$.

To describe the elementary divisors, or what is the same, the invariant factors of $M$, we introduce the following notation. For $1 \leqq r$ $\leqq m$, let $c(r)$ denote the largest integer $l$ such that the submatrix of $M$ consisting of the entries from rows 1 through $r$ and columns $l$ through $m$, has rank $r$. For example, $c(1)$ is the column index of the last nonzero entry of the first row of $M$.

We now indicate a procedure for finding the invariant factors of $M$. Subtract appropriate multiples of column $c(1)$ from columns preceding it so that all entries of the first row except $a_{1, c(1)}$ become 0 . Subtract suitable multiples of the resulting first row from the rows below it so that all entries $(i, c(1)), 2 \leqq i \leqq m$, become 0 . If $c(1)<m$, repeat the process with columns $c(1)+1$ through $m$. These elementary operations transform $M$ into a matrix

1 This research was supported in part by NSF Grant No. GP 4013. 
DECOMPOSITION OF PRODUCTS OF MODULAR REPRESENTATIONS 1013

$$
\left[\begin{array}{ll}
0 & D_{1} \\
M_{1} & 0
\end{array}\right]
$$

where $D_{1}$ is diagonal with each entry $a_{1, c(1)}$. It follows that the first $m-c(1)+1$ invariant factors of $M$ are $t^{n-c(1)+1}$. It can be shown that each entry of $M_{1}$ is the determinant of an appropriate submatrix of $M$, which implies that either the upper right-hand entry of $M_{1}$ is nonzero or else $M_{1}$ has a triangular block of zeros in its upper righthand corner. The procedure may then be repeated with $M_{1}$, leading to the following result.

Theorem. Let the sequence $c(1), c(2), \cdots, c(m)$ be of the form

$$
\begin{aligned}
c(1) & =c(2)=\cdots=c\left(r_{1}-1\right)>c\left(r_{1}\right) \\
& =c\left(r_{1}+1\right)=\cdots>c\left(r_{s}\right)=1
\end{aligned}
$$

and set $r_{0}=1$. Let

$$
e(i)=n-c\left(r_{i}\right)+r_{i}, \quad 0 \leqq i \leqq s .
$$

Let $f_{0}=m-c\left(r_{0}\right)+1$ and for $1 \leqq i \leqq s$,

$$
f_{i}=c\left(r_{i-1}\right)-c\left(r_{i}\right) \text {. }
$$

Then the invariant factors of $M$ are $t^{(i)}$ with multiplicity $f_{i}, 0 \leqq i \leqq s$.

From this result one easily obtains the formulas of Green [1] used to show the semisimplicity of the representation algebra of $K G$.

\section{REFERENCES}

1. J. A. Green, The modular representation algebra of a finite group, Illinois J. Math. 6 (1962), 607-619.

2. W. E. Roth, On direct product matrices, Bull. Amer. Math. Soc. 40 (1934), 461-468.

3. B. Srinivasan, The modular representation ring of a cyclic p-group, Proc. London Math. Soc. (3) 14 (1964), 677-688.

UNIVERSITY OF ILLINOIS 